\title{
Performance of TCP, UDP and SCTP on Sensor Network with Different Data Reporting Intervals
}

\author{
B. Chellaprabha ${ }^{1}$, Dr. S. Chenthur Pandian ${ }^{2}$, Dr. C.Vivekanandan ${ }^{3}$ \\ ${ }^{1}$ (Head, Department of Computer Science and Engineering, SNS College of Engineering, India \\ ${ }^{2}$ (Principal, Dr. Mahalingam College of Engineering \& Technology, Tamilnadu, India. \\ ${ }^{31}$ Dean, Electrical Sciences, SNS College of Engineering, India
}

\begin{abstract}
The performance of the congestion control algorithms of most of the reliable transport protocol of internet, particularly wireless networks and mobile ad-hoc networks, are not satisfactory under a high density sensor network applications, since they are designed mainly for wired networks and not for sensor networks. In this work, we evaluate the performance of some of the popular transport protocols on a congested sensor network scenario. The transport protocols considered for evaluation are Transmission Control Protocol (TCP), User Datagram Protocol (UDP) and Stream Control Transmission Protocol (SCTP). The implementation and the evaluation of these selected protocols in the sensor network is done using Network Simulator (NS2). The performance of these algorithms were measured and analyzed using suitable metrics such as throughput, End-to-End delay, Energy consumption etc..The average performance of the networks is found to be the best in the UDP algorithm based networks, with respect to the five metrics considered in this work. It is also found that TCP provides highest throughput at higher data rate, at long data reporting intervals.
\end{abstract}

Keywords - Congestion Control, Transport Protocols, Sensor Network, TCP, UDP, SCTP

\section{INTRODUCTION}

\subsection{The Wireless Sensor Network (WSN)}

A wireless sensor network is a wireless network consisting of spatially distributed autonomous devices using sensors to cooperatively monitor physical or environmental conditions, such as temperature, sound, vibration, pressure, motion or pollutants, at different locations [1]. Each device in a sensor network is called as Node and each node is normally equipped with wireless communication device, typically a radio transceiver, a microcontroller to channelize the functions of the sensor, and an energy source, usually a battery. The selection of memory size, computational speed, bandwidth, energy and the like are restricted mainly by the cost and size of the nodes.

In general, wireless sensor network transmission is multihop nature and constituted by energy constrained nodes. Since the sensors are usually small and inexpensive and have limited energy sources, any protocols to be deployed in sensor networks need to be aware of energy usage. The data to be transmitted by a sensor node is in the form of packet and the network is equipped with an appropriate routing mechanism that can adapt to the network dynamics. From the viewpoint of a transport protocol, the underneath network is an IP based full functional network. To assure a data packet to be delivered to the destination reliably, a transport layer protocol must be embedded between application and network layer.

High data rate applications involve voluminous data transfer and require a more reliable transmission and hence, persistent congestion may occur [2]. In such high rate sensor network applications a fairly reliable solution is mandatory to avoid congestion and to maintain complete and efficient data transfer between many sources and one or more sinks [3].

The paper is organized in the following manner. Next section discusses the necessity of this work in the present scenario. Chapter II gives an overview of the transport protocols considered paper viz. TCP, UDP and SCTP and an overview on congestion control. Chapter III details the model of WSN considered in this work and the metrics considered to evaluate the performance of the WSN. The simulated responses of the WSN with its parameters set to the values discussed in Chapter III, in terms of the metrics considered in this work, for all the three different protocols, along with an exhaustive analysis, are given in Chapter IV. Chapter $\mathrm{V}$ discusses the inferences arrived out of the analysis.

\subsection{Need of this work}

TCP and SCTP are well suited for efficient streaming communication over unreliable Internet but they need to be improved for better performance over a typical wireless sensor network scenario. A typical wireless sensor network is highly unstable as it is error-prone due to various reasons such as interference of radio signal, radio channel contention, and survival rate of nodes [4]. This error rate is increased significantly in a multi-hop network due to channel contention. Further, in a sensor network error rate is much higher and bandwidth is smaller than that of fixed networks. As a consequence, running conventional TCP or SCTP protocol on a wireless sensor network will potentially suffer from severe performance degradation. 
It is obvious that the capabilities of wireless sensor nodes are much less than that of their fixed network counterparts, due to various reasons [5]. The complexity in implementing a standard TCP or SCTP protocol inside the tiny sensor nodes further degrades the performance of wireless nodes. However, the capabilities of the modern sensor nodes have improved so much to accommodate a fully functional TCP like protocol stack inside them. So, evaluating these classical protocols on wireless sensor networks may provide an opportunity to understand and estimate their performance wireless sensor networks. An in-depth analysis, based on the above study, may help us to include the necessary modifications and improvements so as to accommodate those protocols for wireless sensor networks. More experiments and evaluations are needed to understand the behavior of these protocols under congested sensor network scenario which may lead to a better congestion control algorithm for wireless sensor networking.

\section{The TransPort Protocols AND CONGESTION CONTROL}

\subsection{Transport Layer}

The main objective of the transport layer is to provide reliable and controllable end-to-end communication service for applications with connection-oriented data stream support. Though the transport protocols, such as TCP, work efficiently in infrastructure networks, their performance is relatively poor when employed in wireless sensor networks, hence require considerable modifications [6]. As TCP is strictly end-to-end reliability model, confirmations and retransmissions, if any, need to follow the complete sourceto-destination path, resulting in inefficient use of bandwidth along with energy burden on already energy hungry nodes. The transport protocols proposed so far deal either optimization of a particular parameter or application specific. It is desirable to design a transport layer protocol that can support multiple applications in the same network, provide controlled variable reliability, address congestion issues, reduce latency and maximize throughput [2].

\subsection{Congestion Control}

Congestion, transmitting packets beyond the admissible limit of a link, may not be constant over the different points of the WSN, due to its multi-hop nature and a different degree of congestion might be felt at different points over WSN [7]. It is obvious that the congestion is high around the base station or 'sink', due to the convergent nature of the traffic towards the base station. This huge amount of data flow, along with the constrained buffer size, results in congestion, which may lead to a significant amount of packet loss or data loss. This further necessitates packet retransmission and causes a significant amount of energy loss and delivery delay. High data rates, sudden burst of data and collisions are other reasons of congestion in sensor networks.

Congestion may be sensed by buffer drops and increased delays in traditional networks and researchers have developed end-to-end adaption and network layer dropping or signaling techniques to prevent the network from collapsing due to congestion, over period of time. In addition to buffer overflows, the quality of the WSN is degraded mainly due to the excessive traffic over the radio channel as those channels are not insulated from each other as in the case of wired or provisioned cellular links, resulting in the degradation of the channel quality. The quality of the network is further deteriorated by poor and time varying channel quality, asymmetric communication channels, multi-hop environment etc., [8].

Congestion detection, congestion notification and rateadjusting are the three major phases of Congestion control. The main performance objective of a congestion control protocol is energy efficiency which is achieved by minimizing or avoiding packet loss due to buffer overflow and assuring prolonged life time for the system. Maintaining a fairly reasonable throughput in each node by rate-adjustment i.e. the rate at which the sensors send data to sink node, and packet scheduling is the another major objective of congestion control protocols. Another desirable characteristic of congestion control protocols is to provide a better Quality of Service (QoS) in terms of packet loss ratio, packet delay, throughput etc., [9]. Hence, it is required not only to detect the congestion but also to implement an appropriate avoidance technique to minimize losses and to increase the overall performance of WSN.

\subsection{The Transport Protocols under Evaluation}

\subsubsection{Transmission Control Protocol (TCP):}

A basic strategy for communication among dissimilar networks was suggested by Vint Cerf and Bob Kahn in 1974 and called as Transmission Control Protocol (TCP). The evolution that followed provides a reliable and ordered delivery of a data among the communicating nodes.

Internet protocol suite comprises two core protocols viz. TCP and Internet Protocol (IP) and commonly referred to as $T C P / I P$. In the event of data transmission between two computers, TCP, staying between an application program and the Internet Protocol, provides reliable and ordered delivery of a stream of bytes from a program on one computer to another program on another computer. That is, when an application program desires to send a large chunk of data across the Internet using IP, instead of breaking the data into IP-sized pieces and issuing a series of IP requests, the software can issue a single request to TCP and let TCP handle the IP details.

A packet, a piece of information, is a sequence of octets and consists of a header or the destination address, followed by a body or information. IP works by exchanging packets. Since packet transfer is not reliable, a technique known as positive acknowledgment with retransmission is used to guarantee reliability of packet transfers. This fundamental technique requires the receiver to respond with an acknowledgment message as it receives the data. The sender keeps a record of each packet it sends, and waits for acknowledgment before sending the next packet. The sender also keeps a timer from when the packet was sent, and retransmits a packet if the timer expires. The timer is needed in case a packet gets lost or corrupted. Loss of IP packets 
may occur due to several reasons such as network congestion, traffic load balancing, unpredictable network behavior etc. In addition the packets may be duplicated or delivered out of order. TCP detects these problems, requests retransmission of lost data, rearranges out-of-order data, and minimizes network congestion to reduce the occurrence of the other problems. With TCP as the benchmark, a transport mechanism in WSN should have basic functionalities such as reliable transport of data, better congestion control means, reasonable rate-control and acceptable fairness [10].

\subsubsection{User Datagram Protocol (UDP):}

The User Datagram Protocol (UDP) is another protocol with TCP/IP suit which can send messages, also known as datagrams in this protocol, to other nodes on IP network without requiring prior communications to set up special transmission channels or data paths. The protocol was designed by David P. Reed in 1980 and formally defined in RFC 768[11].

UDP is the simplest form of transmission and there is no handshaking between the transmitting nodes. UDP assumes that error checking and correction is either not necessary or performed in the application, avoiding the overhead of such processing at the network interface level. Due to this, the arrival of datagrams may not be in the same order in which they were transmitted. The quality of service is further deteriorated by data duplication and data loss. UDP is more appropriate for applications where time is precious than data loss and hence, seldom suitable for real-time applications.

\subsubsection{Stream control transmission protocol (SCTP):}

In Stream Control Transmission Protocol (SCTP) the transmission of a message is in one operation and reception of the exact message is also in one operation. "Multihoming" and "Multi-streaming" are the two special features of SCTP and a connection between two endpoints in this context is called an "association".

Multi-homing is defined as the ability of an association to support multiple IP addresses or interfaces at a given end point. Use of more than one address permits re-routing of packets in event of failure and also provide an alternate path for retransmissions, thus resulting in greater survivability of the session. Endpoints can exchange lists of addresses during initiation of the association. One of the addresses is designated as the primary address to receive data. A single port number is used across the entire address list at an endpoint for a specific session. Heartbeat chunks are used to monitor availability of alternate paths with thresholds set to determine failure of alternate and primary paths.

Multi-streaming represents a sequence of messages, either long or short, within a single association and different from multiple streams. The messages include control flags for segmentation and reassembly. Stream Identifiers and Stream Sequence numbers are included in the data packet to allow sequencing of messages on a per-stream basis which eliminates the unnecessary head-of-line blocking between independent streams of messages, in case of loss in any of the streams. In addition SCTP provides mechanism for designating order-of-arrival delivery as opposed to ordered delivery.

\section{Simulation OF SENSOR NETWORK}

The proposed sensor networks have been simulated NS2 simulator. The TCP, UDP and SCTP protocols available under NS2 were used along with their default parameters.

Figure. 1 shows the simulated sensor network field. The node designated as ' 0 ' is the sink node. Sensor nodes 1 to 7 are designed to have higher transmision power (Tx) and act as gate way to sink node and the rest are normal nodes and can transmit only to a short distance.

Fig. 1. The Wireless Sensor Network

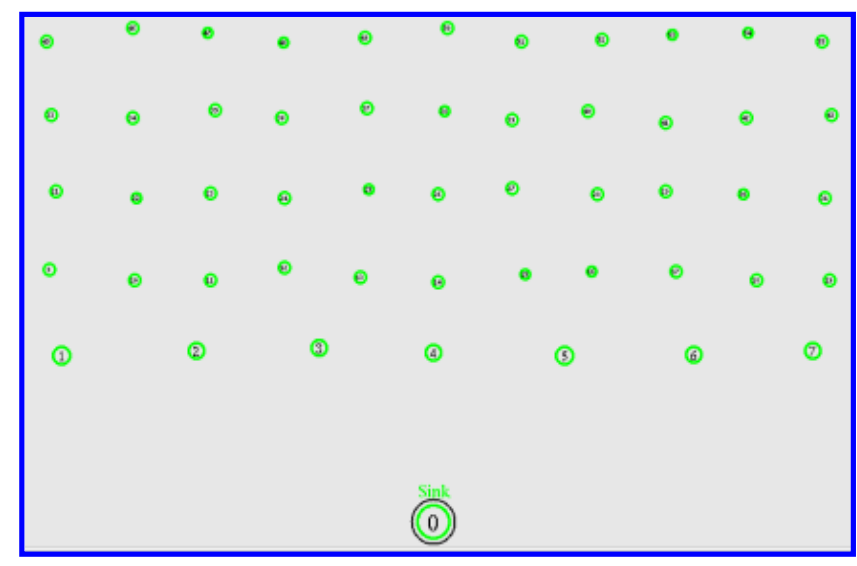

\subsection{Simulation Parameters for Sensor Network}

The capabilities of the nodes used in this simulation are so selected that they are equivalent MICA mote sensors, a widely used nodes by researchers and developers and the selected parameters for the simulation are given in Table 1. Energy model of NS2 is used to study the power drain characteristics of the nodes. The energy parameters are set according to the node capability.

\subsection{Metrics considered for Evaluation}

3.2.1. Throughput: The rate of data packet arrival with respect to time at the destination or sink is the throughput of the network and hence, higher the rate is better the congestion control algorithm.

3.2.2. Energy Consumption: The average energy consumed by all the nodes of the network is considered as a metric to assess the performance of the congestion control algorithms. The energy consumption of a node depends on several parameters such as sensor data reporting interval, routing protocol, transport protocol, congestion algorithm of the transport protocol etc., and it is obvious that the lower energy consumption signifies better congestion control algorithm.

3.2.3. Routing Load: It is the number of routing packets required to transmit a data packet successfully to the sink node. A better congestion algorithm provides a relatively lower routing load for the given data packet.

3.2.4. Mac Load: MAC load means the average number of MAC messages generated to each data packet successfully 
delivered to the destination. Hence, lower MAC load signifies better congestion control algorithm.

3.2.5. Dropped Packets: The data packets that fail to reach sink due to congestion during transmission are dropped packets. The number of dropped packets, with respect to the change in sensor reporting interval and the average of dropped packets at all nodes are considered in this work. In a better congestion control algorithm the count of dropped packets is significantly low.

3.2.6. End to End Delay (E2E) : EED is the cumulative delay that might come about as a result of buffering during discovery of routes over sensor network, queuing at interfaces of the sensor nodes, delays in retransmission at the MAC, and the time taken for propagation and transfer over the sensor field.

$$
\mathrm{E} 2 \mathrm{E}=\sum_{n=1}^{W}\left(x_{n 2}, s_{n}\right)
$$

where

-the time that data packet $\mathrm{n}$ was sent

-the time that data packet $\mathrm{n}$ was received

and - the total number of data packets received

Table 1 : Parameters of the Sensor node and Network

\begin{tabular}{lll}
\hline Parameter & & Value \\
\hline Transmission Range & & \\
Sink Node & $:$ & $\mathbf{1 5 0} \mathbf{~ m}$ \\
Sensor Node 1 to 7 & $:$ & $\mathbf{1 5 0} \mathbf{~ m}$ \\
Other Sensor Nodes & $:$ & $\mathbf{6 0} \mathbf{~ m}$ \\
Channel & $:$ & Wireless Channel \\
Propagation & $:$ & Two Ray Ground \\
Physical Medium & $:$ & Wireless Physical \\
Antenna & $:$ & Omni Antenna \\
Routing Protocol & $:$ & AODV \\
Mac Type & $:$ & $\mathbf{8 0 2 . 1 1}$ \\
Queue & $:$ & DropTail/PriQueue \\
Queue Size & $:$ & $\mathbf{5 0}$ \\
Sensor Reporting Interval & $:$ & $\mathbf{1 , 2} \mathbf{5 , 1 0}$ and 20 sec \\
Traffic Application & $:$ & CBR \\
Sensor Data Size & $:$ & $\mathbf{2 5 6}$ bytes \\
Number of Nodes & $:$ & $\mathbf{5 6}$ \\
Topographical Area & $:$ & $\mathbf{8 0 0 m} \mathbf{x}$ 400m \\
Transport Protocols & $:$ & TCP, UDP, SCTP \\
Simulation Time & $:$ & $\mathbf{1 0 0 ~ s e c}$ \\
Node Receiving & $:$ & $\mathbf{3 . 6 5 2 e}-10$ \\
Threshold & $:$ & $\mathbf{2 . 4 e 0 9 ~ H z}$ \\
Node Signal Frequency & & \\
\hline
\end{tabular}

\section{RESULTS AND ANALYSIS}

Wireless sensor network with parameters discussed in section III was simulated, with three different transport protocols TCP, UDP and SCTP separately, over a wide range of sensor data reporting intervals. The results obtained are tabulated and plotted, various analysis were made to estimate the performance of the networks based on the metrics considered in this work and the inferences arrived are discussed in the following section.

Figure 2 shows the performance of the three transport protocols in terms of throughput with respect to different sensor data reporting intervals. It may be observed from the plot that at low intervals the performance of UDP protocol is far better than that of other two protocols and TCP follows it. As the time interval increases the differences in the throughput among the protocols converges with TCP overtaking UDP. However, UDP is found to deliver best performance when average throughput over a wide range of time period is considered, as shown in Figure 3. It may also be observed that the average throughput of TCP protocol closely follows UDP, where as the performance of SCTP is relatively very low.

The amount of energy consumed by the network with different protocols over same time interval is plotted in Figure 4. It is found that the energy consumption rate is high at low time intervals, falls at a faster rate as time interval increases and almost same at high time intervals for all the three protocols.

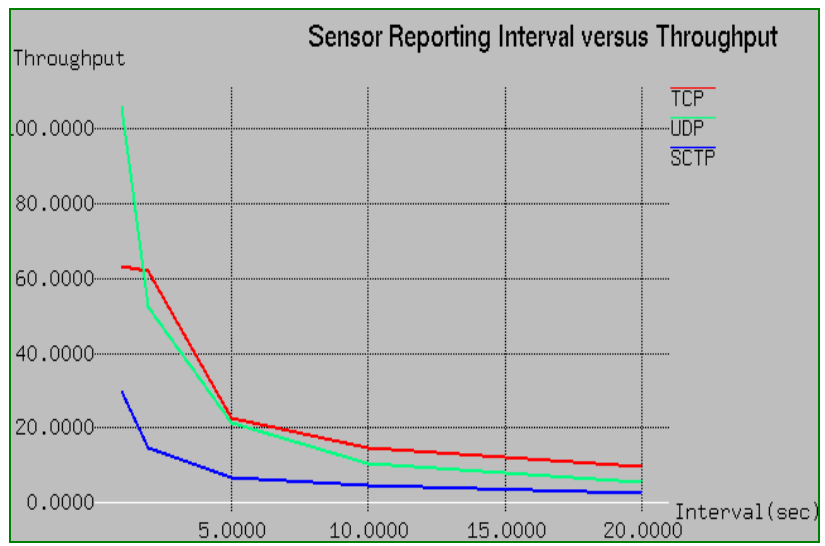

Fig 2 : Throughput with respect to Different Data Interval

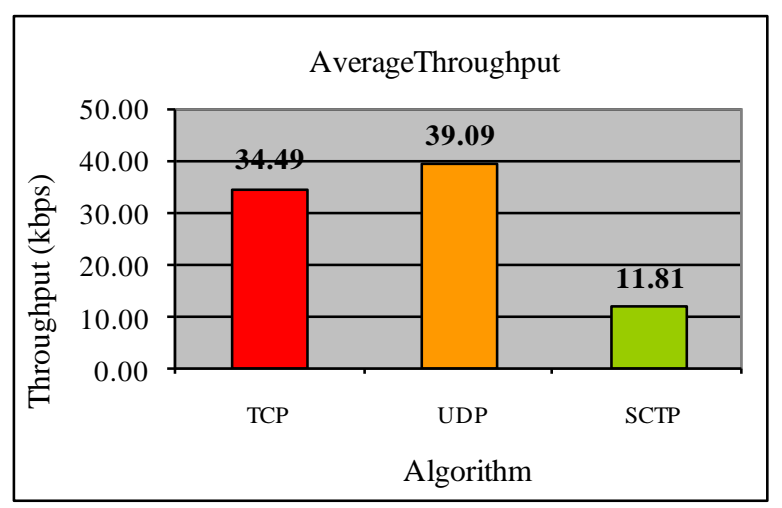

Fig 3 : The Average Throughput

It is observed that, though UDP protocol consumes high energy during low time intervals, the consumption is the lowest during the high time intervals. The remaining two protocols consume almost same energy over the time period considered, barring the initial time interval.

Considering the average energy consumption over the same time period it is found that the UDP is taking the least 
power, despite its initial high consumption and followed by other two protocols, with almost same energy consumption as shown in Figure 5.

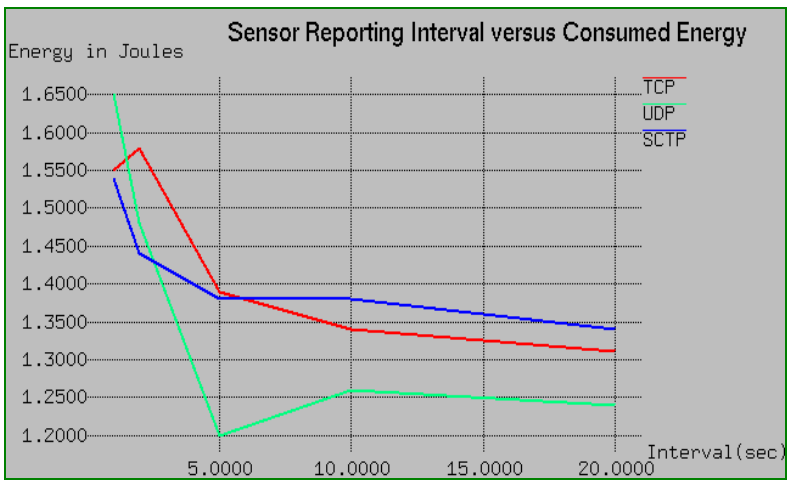

Fig 4 : Energy Consumed with respect to Different Data Interval

The performance based on the routing load metric is considered next and its changes with respect to the time interval, for all the three protocols, are as shown in Figure 6. The performance of the UDP protocol is found to be superior over the entire time period considered. The performances of TCP and SCTP follow UDP with the additional routing load of approximately $80 \%$ and $85 \%$ respectively.

Figure 7 shows the average routing load over the time interval. Obviously the loading effect with respect to UDP is very low and standing at 8.66 where as TCP and SCTP at 56.45 and 82.00 respectively, proving that UDP is best suited protocol with respect to routing load metric.

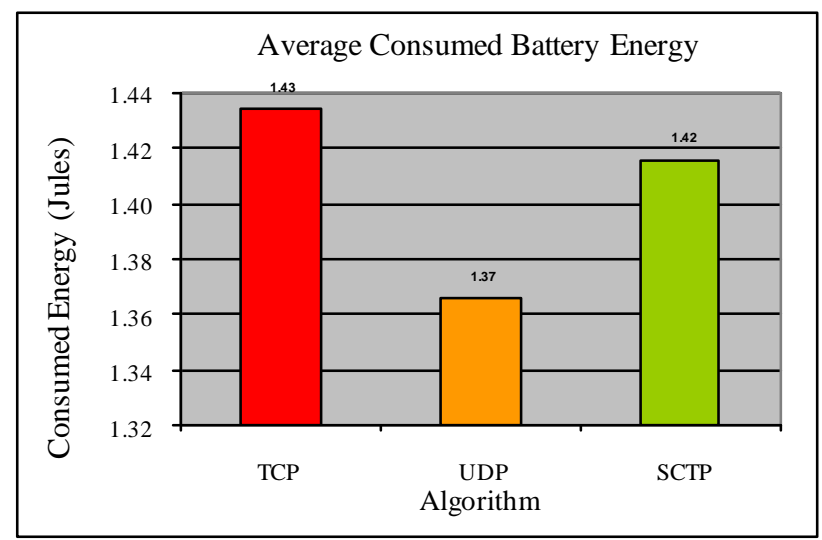

Fig 5 : The Average Consumed Energy

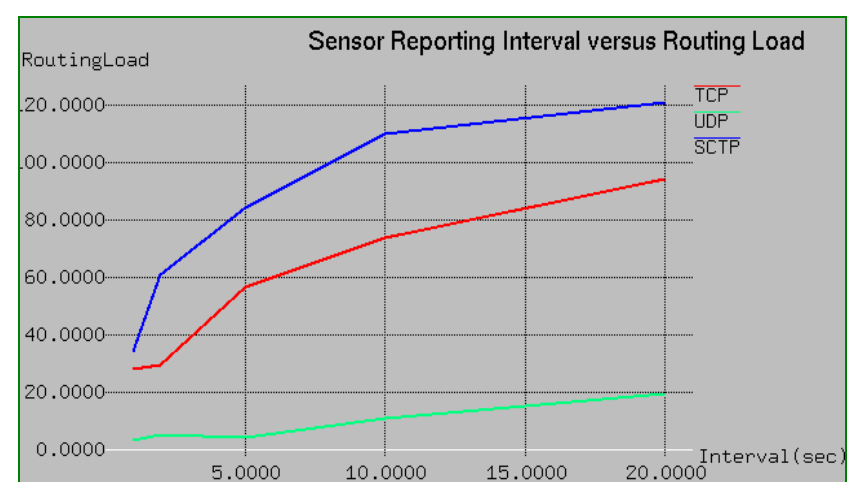

Fig 6 : Routing Load with respect to Different Data Interval

Average Routing Load

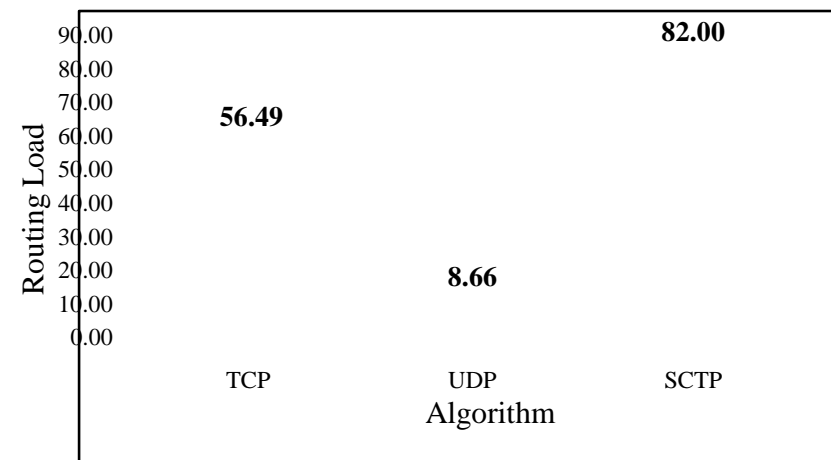

Fig 7 : The Average Routing Load

From Figure 8, which connects the sensor data reporting intervals with the corresponding MAC loads for all the three protocols considered in this paper, it is evident that the networks with UDP outperform the remaining two. This factor is further augmented when the average MAC load over the same time period is considered, since, the average load is significantly low in case of UDP upon comparing TCP and SCTP, as shown Figure 9.

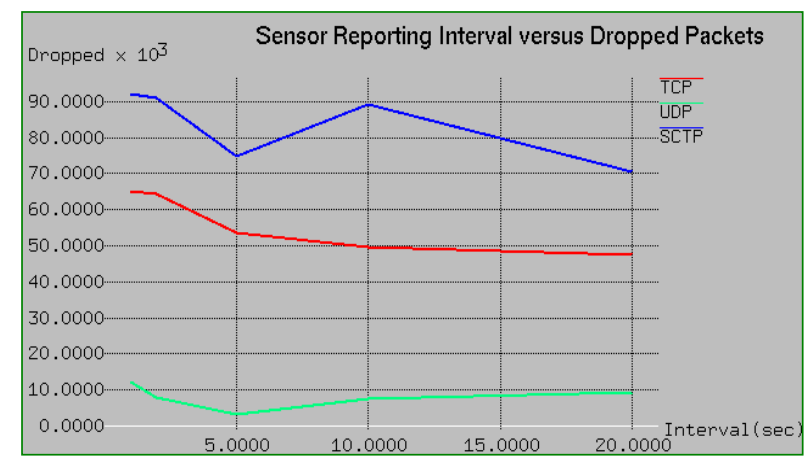

Fig 8. Mac Load with respect to Different Data Interval 


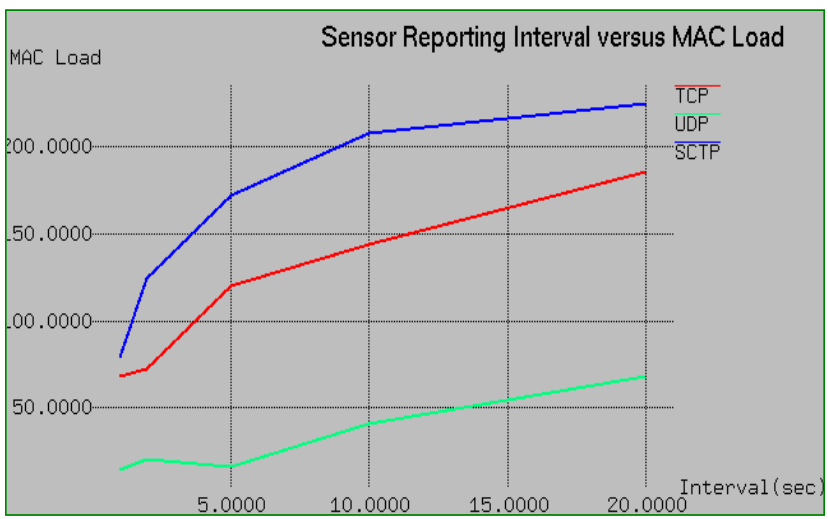

Figure 9: The Average MAC Load

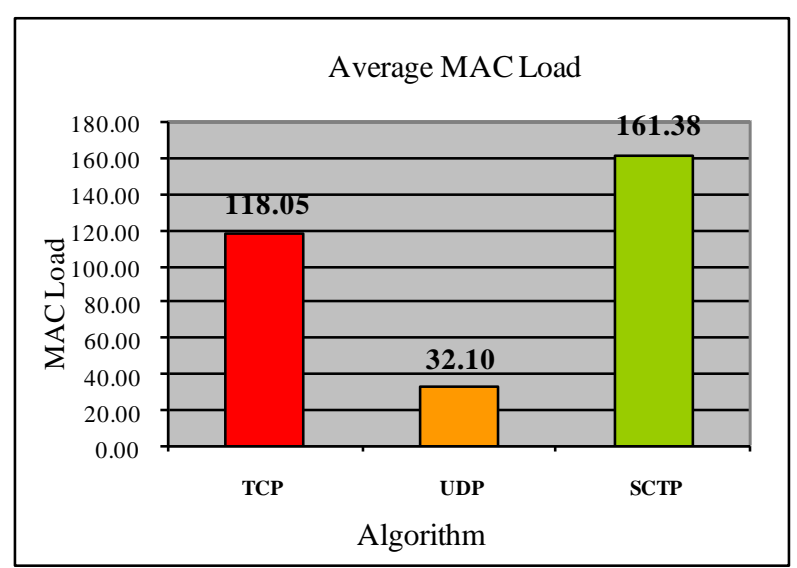

Figure 10 : Packets Dropped with respect to Different Data Interval

Considering the amount of dropped packets, one of the major issues in wireless sensor networks, the system employed with UDP outplays the rest with a relatively low packet loss as shown in Figure 10. From the plot it may be observed that the amount of dropped packets is very low and almost constant irrespective of the time interval in UDP. As far as SCTP protocol is considered, it exhibits greater variations over the time period considered and the number of dropped packets is always high among all the three protocols, where as the TCP shows almost similar performance to that of UDP and lie between the performances of SCTP and UDP and closer to SCTP. Figure 11 shows the average packet drop over the time period, which conveys that the packet drop prohibitively higher with SCTP, closely followed by TCP and almost negligible with UDP, revealing that the UDP is delivering the best performance.

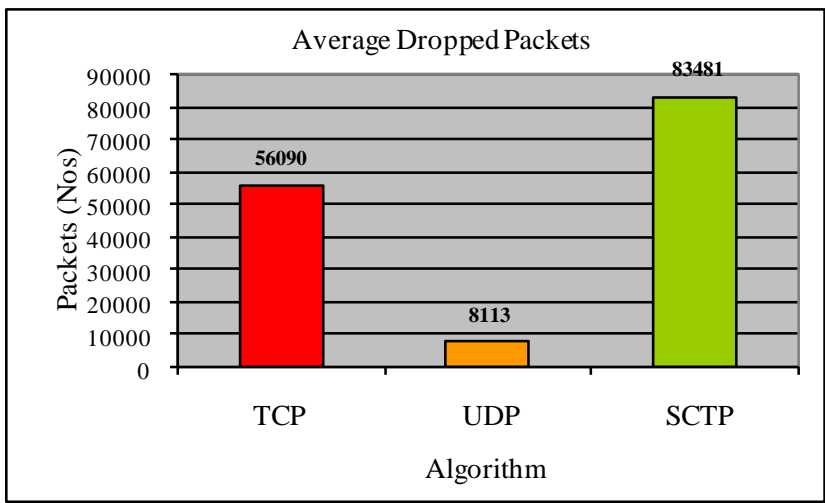

Figure 11: The Average Dropped Packets

Over the selected data reporting interval, the fluctuations in End-to-End delay (E2E) are very high in case of wireless sensor networks employed with TCP and SCTP and higher in case of TCP, as shown in Figure 12. From the same figure it may be noticed that the E2E delay in networks employed with UDP is very low and in fact negligible, comparing the performances of other two protocols. The supremacy of the UDP with respect to E2E delay is clear, considering the average delay over the stipulated time period as shown in Figure 13.

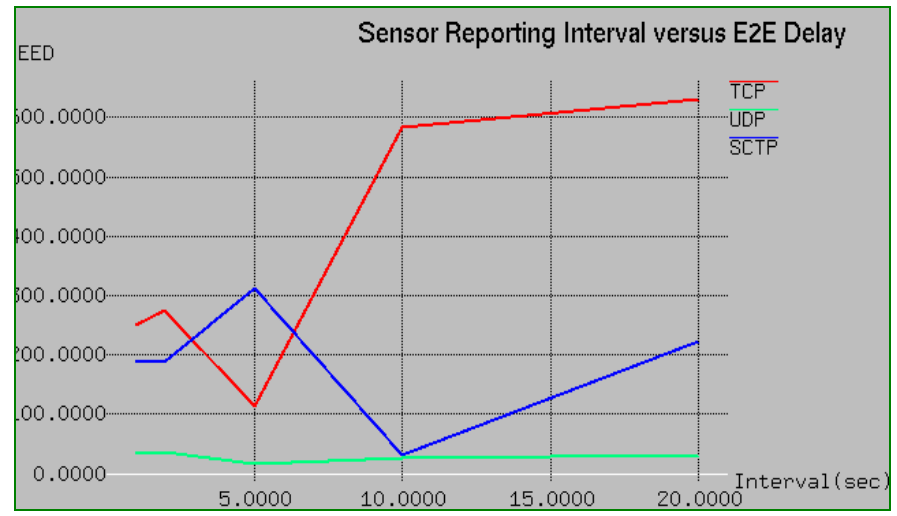

Figure 12 : The End to End Delay with respect to Different Data Interval

Average End to End Delay

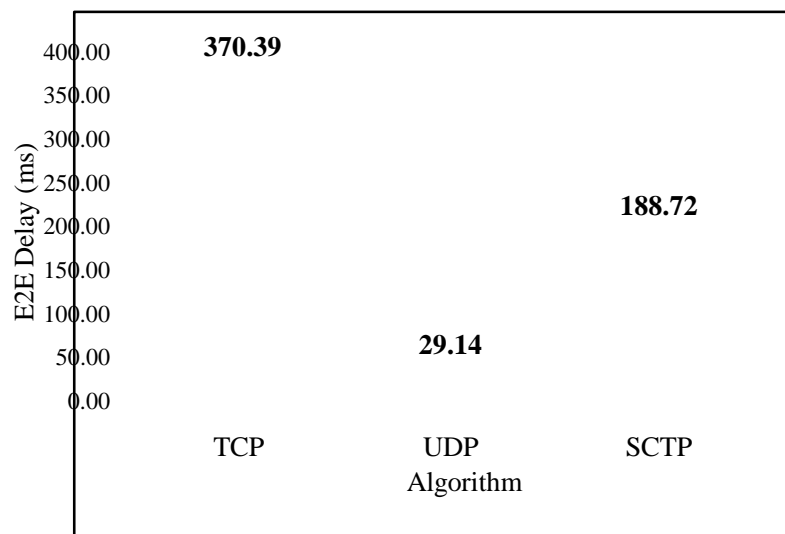

Figure 13 : The Average End to End Delay 


\section{CONCLUSION}

In this paper an exhaustive study on the congestion control in wireless sensor networks using three protocols viz. TCP, UDP and SCTP in transport layer, based on the five metrics, has been done. The performance of the networks, simulated using NS2, based on the desired metrics over a range of data reporting interval has been presented.

Though the throughputs of all the three protocols are almost same at low data intervals, TCP is marginally better at high data intervals. However, it was also found that the average throughput of UDP is having an edge over its other two counterparts. It was also estimated that wireless sensor networks employing UDP are most reliable as the data packet loss is negligible comparing other two protocols. This is due to the relatively lesser load on the network in terms of MAC load and routing load. All this is achieved with least energy consumption and relatively negligible E2E delay, which further enhances the productivity of the UDP. Hence, it may be concluded that the performance of wireless sensor networks employing UDP found to be the best among the three protocols considered in this paper.

\section{ANNEXURE}

Table 2, Table 3 and Table 4 show the values obtained with respect to different metrics considered here over the desired data reporting intervals, respectively for TCP, UDP and SCTP. The observation is made at shorter time intervals during low data reporting time periods as the rate of change in the metrics considered is higher. The same is observed at longer time intervals during high data reporting intervals due to relatively lesser rate of change in the parameters. The data intervals in the tables are scaled down by the factor for better readability.

Table 2 : Performance of TCP

\begin{tabular}{|c|c|c|c|c|c|c|}
\hline 苐 & 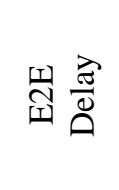 & 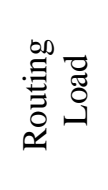 & 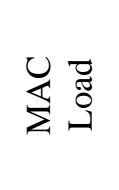 & 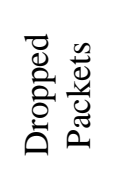 & 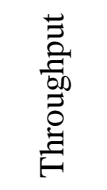 & 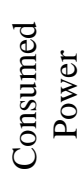 \\
\hline 1 & 249.01 & 28.32 & 68.27 & 65052 & 63.19 & 1.55 \\
\hline 2 & 275.87 & 29.37 & 72.74 & 64538 & 62.09 & 1.58 \\
\hline 5 & 114.08 & 56.72 & 120.09 & 53616 & 22.71 & 1.39 \\
\hline 10 & 583.10 & 73.64 & 143.74 & 49793 & 14.59 & 1.34 \\
\hline 20 & 629.88 & 94.40 & 185.42 & 47450 & 9.88 & 1.31 \\
\hline Avg & 370.39 & 56.49 & 118.05 & 56090 & 34.49 & 1.43 \\
\hline
\end{tabular}

Table 3 : Performance of UDP

\begin{tabular}{|c|c|c|c|c|c|c|}
\hline 苞苞 & 푐 & 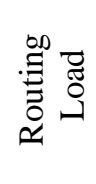 & 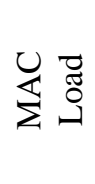 & 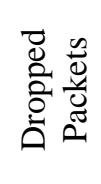 & 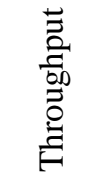 & 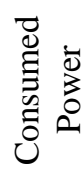 \\
\hline 1 & 34.47 & 3.56 & 14.87 & 12323 & 105.7 & 1.65 \\
\hline 2 & 34.89 & 5.09 & 20.42 & 8025 & 52.24 & 1.48 \\
\hline 5 & 16.99 & 4.14 & 16.12 & 3301 & 21.56 & 1.20 \\
\hline 10 & 27.19 & 11.01 & 40.96 & 7654 & 10.52 & 1.26 \\
\hline 20 & 32.17 & 19.51 & 68.12 & 9263 & 5.43 & 1.24 \\
\hline Avg & 29.14 & 8.66 & 32.10 & 8113 & 39.09 & 1.37 \\
\hline
\end{tabular}

Table 4 : Performance of SCTP

\begin{tabular}{|c|c|c|c|c|c|c|}
\hline 悉 & 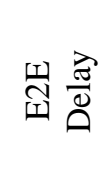 & 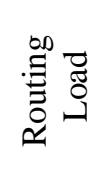 & 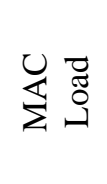 & 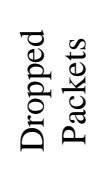 & 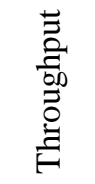 & 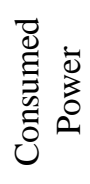 \\
\hline 1 & 189.17 & 34.21 & 79.32 & 92022 & 29.58 & 1.54 \\
\hline 2 & 188.42 & 61.03 & 124.16 & 91193 & 14.89 & 1.44 \\
\hline 5 & 312.48 & 84.41 & 171.60 & 74672 & 6.90 & 1.38 \\
\hline 10 & 30.95 & 109.72 & 207.37 & 89088 & 4.90 & 1.38 \\
\hline 20 & 222.57 & 120.62 & 224.44 & 70430 & 2.77 & 1.34 \\
\hline Avg & 188.72 & 82.00 & 161.38 & 83481 & 11.81 & 1.42 \\
\hline
\end{tabular}

\section{REFERENCES}

[1] Jang-Ping Sheu, Li-Jen Chang and Wei-Kai Hu, "Hybrid Congestion Control Protocol in Wireless Sensor Networks", Journal Of Information Science And Engineering 25, pp. 1103-1119, 2009.

[2] Y. G. Iyer, S. Gandham, S. Venkatesan, "STCP: A Generic Transport Layer Protocol For Wireless Sensor Networks", 14th International Conference on In Computer Communications and Networks, pp. 449-454, 2005.

[3] Alam and Hong, "Congestion-Aware and RateControlled Reliable Transport in WSNS", The Institute of Electronics, Information and Communication Engineers, 2009.

[4] Yao-Nan Lien, "Hop-by-Hop TCP for Sensor Networks", International Journal of Computer Networks \& Communications - IJCNC, Vol.1, No.1, April 2009.

[5] Jang-Ping Sheu1, Li-Jen Chang And Wei-Kai Hu, "Hybrid Congestion Control Protocol in Wireless Sensor Networks", Journal of information science and engineering 25, 1103-1119,2009.

[6] Paulo Rogério Pereira, et.al. "End-To-End Reliability in Wireless Sensor Networks: Survey and Research Challenges", EuroFGI Workshop on IP QoS and Traffic Control P. Pereira (Ed.)' Lisbon, Portugal, December 67, 2007. 
IOSR Journal of Engineering

Apr. 2012, Vol. 2(4) pp: 621-628

[7] Md. Abdur Rahman, Abdulmotaleb El Saddik and Wail Gueaieb, "Wireless Sensor Network Transport Layer: State of the Art", S.C. Mukhopadhyay, R.Y.M. Huang (eds.), Sensors, 221, Springer-Verlag Berlin Heidelberg, 2008.

[8] B. Hull, K. Jamieson, and H. Balakrishnan, "Mitigating congestion in Wireless Sensor Networks", SenSys '04: Proc. 2nd International Conference on Embedded Networked Sensor Systems, pp.134-147, ACM, 2004.

[9] Chonggang Wang1, Kazem Sohraby, Victor Lawrence, Bo Li, Yueming $\mathrm{Hu}$, "Priority-Based Congestion Control in Wireless Sensor Networks", Proceedings of the IEEE International Conference on Sensor Networks, Ubiquitous, and Trustworthy Computing - SUTC'06, 2006.

[10] Xiaohua Luo, Kougm Zheng, Yunhe Pan, Zhaohui Wu, "A TCP/IP implementation for wireless sensor networks", IEEE International Conference on Systems, Man and Cybernetics, 2004.

[11] J. Postel, User Datagram Protocol RFC: 768,1980.

[12] Maria-Dolores Cano, "On the Use of SCTP in Wireless Networks", Recent Advances in Wireless Communications and Networks, ISBN 978-953-307274-6 Edited by: Jia-Chin Lin Publisher: InTech, pp.245-266, 2011. 